\title{
Leather Quality Estimation Using an Automated Machine Vision System
}

\author{
Parag Kohli and Ms. Shalvi Garg \\ ${ }^{1}$ (ECE Deptt., BGIET Sangrur, Punjab INDIA, ${ }^{2}$ (Asst. Professor ECE Deptt. BGIET Sangrur, Punjab INDIA,
}

\begin{abstract}
In the presented work, it is proposed to design the feature vector of the parameters of leather material in order to completely define the quality of the leather material. The proposed parameters are holes, cracks, spots, cuts, and roughness etc.. The defects are localized according to their position on leather surface, their size and shape. The histogram analysis method is proposed to be developed for use with very low-level image features, such as color and luminance and is used as an image descriptor for color-matching requirements. In the present scenario, it is observed that the leather quality is highly sensitive to surface finish of the leather material. Manually it is not possible always to inspect each area of the leather surface under test because of heavy lot of material and it is time consuming too. The main problem during the inspection is that how to achieve the repeatable quality at regular interval. Therefore, in order to get the authentic leather quality, it is required to have a set of features that could be given some numerical value so that the quality can be justified in quantified manner. A machine vision system offers a fair solution in order to solve this problem. In the proposed work, we propose to deduce some mathematical parameters besides size and location that are arranged in a vector so as to determine the leather quality.
\end{abstract}

Keywords: GLCM $\rightarrow$ Gray Level Co-occurrence Matrix

\section{INTRODUCTION}

Automated visual inspection of leather surface defects is very important in the manufacturing of leather products that require unusually high quality. These operations are currently performed by human inspectors who tend to miss considerable numbers of defects because human beings are basically inconsistent and inappropriate for such simple and repetitive tasks. Furthermore, since manual inspection is slow and labor-intensive tasks, they can become a critical bottleneck in the entire production process. In this study we use machine vision to substitute for human inspectors for automated surface inspection. Automated inspection can reduce human workloads and labor costs while increasing throughput. More importantly, higher accuracy can be achieved by eliminating human error due to fatigue.

Leather as a natural material with its variety of visual appearances - non-homogeneous in color, thickness, brightness, wrinkledness, etc. is a complex object for control and analysis. The presence of defects is critical factor for adjustment of the leather for manufacturing of particular good, because the existence of areas with leather defects maybe considered as unusable or useful only for particular purposes. There are well known different methods for analysis of leathers surface defects and classifications [3,4]. The method is based on statistical analysis of the image gray levels. Most of algorithms to detect the leather defect by extracting a set of textural features using co-occurrence matrix approaches[2], the Fourier transform, the Gabor transform[5] and the wavelet transform[1]. These analysis methods are generally based on the extraction of textural features in the spatial and spectral domains, besides high-dimensional feature space, the most difficult task of the featureextraction approach is to choose adequate textural features which can sufficiently represent the uniqueness of the texture in the image.

\section{RELATED WORKS}

Great efforts have been made by scientific and technical community to automate many leather manufacturing processes by using Machine Vision (MV) systems, especially for leather inspection and classification, for nesting, cutting and gluing processes. A machine-vision-based approach for grading leather hides for footwear industry is proposed in [1]; tools for automatically inspecting leather surfaces have been proposed so far in [2 - 7] with the aim of detecting scars, mite nests, warts, open fissures, healed scars, holes, pin holes, and fat folds. Such features are investigated, by using statistical or other computing techniques (e.g. neural networks (NNs), fuzzy systems (FSs) and support vector machines (SVMs)) $[8,9]$. LASER and water jet cutting machines are widely employed [10]; generally speaking, such machines comprise a table where the leather is arranged and a cutting station provided with the cutting device. Both systems may be, substantially, defined as CNC machines which process $2 \mathrm{D}$ leather CAD sketches providing the proper cut path. These systems overcome the limitations of classical leather cut, usually performed by means of pneumatic presses which set on metallic dies manually positioned by the operators. Some systems have also been devised in order to help 
manual edge dyeing; such systems distribute the ink on a cylinder so as to provide, steadily, the right proportion of ink while the operator properly move the leather patch.

\section{METHODOLOGY}

The main steps of the method for leather quality determination realized by the proposed system are as follow:

1. Image grabbing and Thresholding

2. Segmentation of defects

3. Computation of Centre of Gravity of segmented defect - Location Identification

4. Defects area computation

5. Perimeter Estimate

6. Histogram Analysis - Uniformity of Color/surface smoothness estimation

\section{IMAge Capturing AND PReProcessing}

Leather Surface images are captured using the CCD camera to cover a fixed area defined by distance of the camera from the leather surface. Grabbed images are in jpeg format i.e. 24 color bit format and re converted to gray scale color format using rgb2gray command in matlab. Following figure show the result of image preprocessing operations:

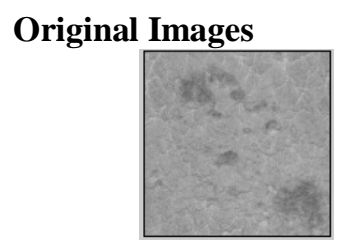

Fig. 1

\section{Histogram Equalized Images}

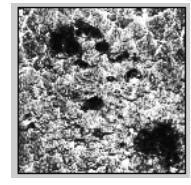

Fig. 2

The gray scale image is now exposed to Otsu algorithm for binarization. A threshold is selected based on minimum class variance. Based on the threshold, the gray scale image is converted to black and white image i.e. binary image.

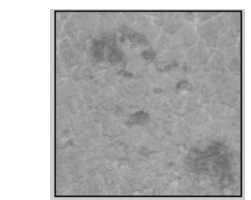

Gray Image

Fig. 3

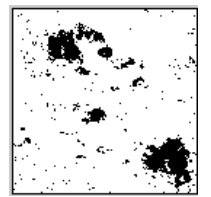

Binary Image

Fig. 4

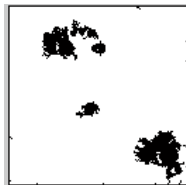

after Noise Removal

Fig. 5

V. Segmentation Of Defects

The binary image is now brought under the segmentation process. Here, the defects are segmented and plotted in different frames of the same size as that of the original. The segmentation is performed using the bwlabel command in matlab. The segmented images are given below:

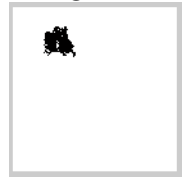

Fig. 6

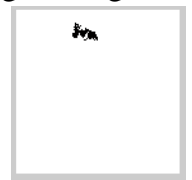

Fig. 7

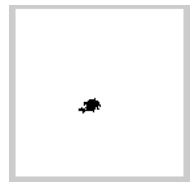

Fig. 8

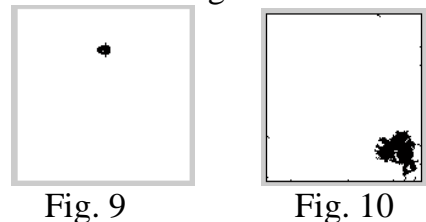

\section{Computation Of Centre Of Gravity Of Segmented Defect - Location IDENTIFICATION}

The centre of gravity of the segmented image is computed by using the following formula:

And

$$
\begin{aligned}
& \mathrm{G}_{\mathrm{x}}=(1 / \mathrm{N}) \sum \mathrm{X}_{\mathrm{i}} \\
& \mathrm{G}_{\mathrm{y}}=(1 / \mathrm{N}) \sum \mathrm{Yi}_{\mathrm{i}}
\end{aligned}
$$


Where $\left(\mathrm{X}_{\mathrm{i}}, \mathrm{Y}_{\mathrm{i}}\right)$ are the coordinates of the pixels on surface. The centre of gravity $\left(\mathrm{G}_{\mathrm{x}}, \mathrm{G}_{\mathrm{y}}\right)$ indicates the location of defect on surface.

\section{DEFECTS AREA COMPUTATION}

The area of defect in segmented image is computed by counting the no. of pixels on the body of the defect. The segmented image is basically the binary image with white as background and black as the defect. Therefore, the total no. of black pixels gives the estimate of the area of the defect on the surface.

\section{Perimeter Estimate}

For computing the perimeter of the surface defect, the contour of the defect is identified. A robert's operator is used for extraction of the contour of the defect. All the contour pixels are marked with different gray shades other than that of the background and object under consideration. If the contour pixel color is $\mathrm{C}$, then no. pixels bearing the color shade $\mathrm{C}$ will be the perimeter of the defect on the surface.

\section{HISTOGRAM ANALYSIS - UNIFORMITY OF COLOR/SURFACE SMOOTHNESS ESTIMATION}

The histogram analysis gives the idea about the smoothness of the leather surface. If the histogram is a uniformly biased towards any side of the gray area, then it may be assumed that the leather surface is smooth and fair one. However, a scattered histogram shows the coarseness of the surface. The degree of smoothness may be computed by measuring the entropy of the image. Higher the entropy, more are the shades scattered on the surface or more coarse is the leather surface and vice versa.

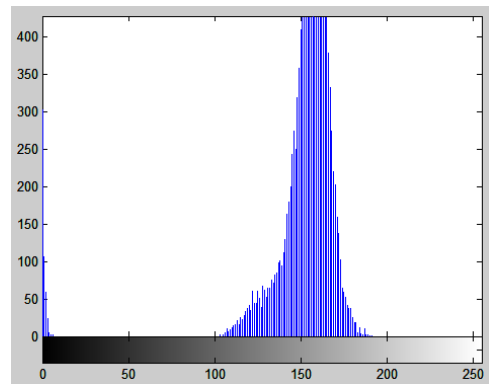

\section{RESULTS}

A leather image is acquired using the CCD camera as shown in fig. 1. The table below shows the results after implementing the algorithm in matlab version 7.5. The table shows results for one of the leather images. However, the algorithm has been tested on approx. 100 test images.

\begin{tabular}{|c|c|c|c|c|}
\hline $\begin{array}{c}\text { IMAGE } \\
\text { No. }\end{array}$ & $\begin{array}{c}\text { LOCATION } \\
(\mathrm{COG})\end{array}$ & AREA & PERIMETER & $\begin{array}{c}\text { DEGREE OF } \\
\text { SMOOTHNESS }\end{array}$ \\
\hline 6 & $(23,32)$ & 123 & 54 & \\
\hline 7 & $37,19)$ & 48 & 35 & \multirow{2}{*}{ ENTROPY $=$} \\
\cline { 1 - 2 } 5 & $100,145)$ & 45 & 31 & \\
\hline 9 & $(34,38)$ & 34 & 21 & \\
\hline 10 & $(200,239)$ & 195 & 120 & \\
\hline
\end{tabular}

\section{CONClusion}

The proposed work presents a data set that can be prepared based on particular application of leather material e.g. leather in wallet industry, shoe industry, belts and baggage industry etc. The smoothness and other parameters value will vary according to their application. In practice, it is required to have a data set of reference leather quality material/surface, and the test image parameters may be computed using the same algorithm and can be compared. The comparison score then may used as to estimate the quality of the leather material to that of the referenced material.

\section{REFERENCES}

[1] Flores M., R. Longhi, M. Albertoni, B. Ferroni. Mastrit Skin 2002 Quality Standard and Global Concept. IPLnet Workshop'02, 2002.

[2] Hoang K., A. Nachimuthu. Image Processing Techniques for Leather Hide Ranking in the Footwear Industry. Machine Vision and Applications, 9, pp. 119-129, 1996.

[3] Krastev K., L. Georgieva. Identification of Leather Surface Defects using Fuzzy Logic. International Conference on Computer Systems and Technologies, IIIA.12, 2005.

[4] Krastev K., V. Dimitrov, L. Georgieva. A Software Program for Leather Quality Estimation. Scientific Session of RU'05, 2005.

[5] United States Hide, Skin, \& Leather Association. Standards Governing the Export of North American Cattle Hides. http://www.ushsla.org, 2005. 
[6] Yeh C., D. B. Perng. Establishing a Demerit Count Reference Standard for the Classification and Grading of Leather Hides. The International Journal of Advanced Manufacturing Technology, 18, pp. 731-738, 2001

[7] K. Hoang and A. Nachimuthu, Image processing techniques for leather hide ranking in the footwear industry, Machine Vision and Applications Volume 9, Number 3, 1996, pp. 119-129.

[8] A. Branca, M. Tafuri, G. Attolico and A. Distante, Automated system for detection and classification of leather defects, Optical Engineering, 1996, Vol. 35, Issue 12, pp. 3485-3494.

[9] K. Hoang, W. Wen, A. Nachimuthu and X.L. Jiang, Achieving automation in leather surface inspection, Computers in Industry, 1997, Vol. 34, Issue 1, pp. 43-54

[10] H. Fuqiang, W. Wen and C. Zichen, 2006, Automatic visual inspection for leather manufacture. Key Engineering Materials, 2006, Vol. 326-328 Issue 1, pp 469-472

[11] Yeh, D.B. Perng, Establishing a Demerit count reference standard for the classification and grading of leather hides, International Journal of Advanced Manufacturing Technology, 2001, Vol. 18, Issue 10, pp 731-738

[12] M.M. Neto, O. Vendrametto and J.P.A. Fusco, Automated System for Leather Inspecti on, The Machine Vision, Emerging Solutions For Future Manufacturing Systems, 2005, Vol. 159, pp. 387-396 